\title{
The Spectral Radius for a Class of Double-Star-Like Tree Systems with Maximal Degree 4
}

\author{
Yufeng Mao, Meijin Xu, Xiaodong Chen, Yan-Jun Liu, and Kai Li \\ Science College, Liaoning University of Technology, Jinzhou, Liaoning 121001, China \\ Correspondence should be addressed to Meijin Xu; xumeijin65@126.com
}

Received 5 September 2015; Revised 19 October 2015; Accepted 21 October 2015

Academic Editor: Weiguo Xia

Copyright ( 2015 Yufeng Mao et al. This is an open access article distributed under the Creative Commons Attribution License, which permits unrestricted use, distribution, and reproduction in any medium, provided the original work is properly cited.

\begin{abstract}
We mainly study the properties of the 4-double-star-like tree, which is the generalization of star-like trees. Firstly we use graft transformation method to obtain the maximal and minimum extremal graphs of 4-double-star-like trees. Secondly, by the relations between the degree and second degree of vertices in maximal extremal graphs of 4-double-star-like trees we get the upper bounds of spectral radius of 4 -double-star-like trees.
\end{abstract}

\section{Introduction}

In recent years, there are a lot of the results of the upper and lower bounds on the spectral radius of graphs. For example, Ellingham and Zha [1] proposed upper bound on the spectral radius of graphs embeddable on a given compact surface; Wang et al. [2] proved that $k$ trees have the second and the third largest spectral radius; Gong [3] discussed some sharp lower bounds for spectral radius of connected graphs and gave a new lower bound for spectral radius; Yuan and Shu [4] gave upper bound for the spectral radius of Halin graphs; Zhou and $\mathrm{Xu}$ [5] showed the upper bounds for the Laplacian spectral radius of a graph by using the CauchySchwarz inequality; Song and Wang [6, 7] got the upper bound of the Laplacian spectral radius of some trees by grafting transformation.

Star-like tree and its generalization $m$-star-like tree are special trees and widely used in computer science. There are a lot of results on the bounds of spectral radius of star-like trees and $m$-star-like trees. For example, Patuzzi et al. [8] gave upper bounds of the spectral radius in star-like trees and double brooms; Aalipour et al. [9] obtained upper bound of double-star-like trees by Laplacian spectral; $\mathrm{Wu}$ and $\mathrm{Hu}$ [10] gave new upper bound of the spectral radii of $m$-star-like trees.

Though many researchers obtained the bounds of spectral radius of star-like trees and $m$-star-like trees, few used the extremal graphs of the two kinds of trees to get the bounds. In this paper, we mainly use graft transformation method to obtain the maximal and minimum extremal graphs of 4-double-star-like trees. Then by the relations between the degree and second degree of vertices in maximal extremal graphs of 4-double-star-like trees we get the upper bounds of spectral radius of 4-double-star-like trees. Ours result provide a new method to research the bound of spectral radius of $m$ star-like trees. By this new method, we may get the second largest index, the third largest index, or the $k$ th largest index of $m$-star-like trees, which provide a valuable tool for further research on $m$-star-like trees.

\section{Preliminaries}

In this paper, all graphs considered here are simple and undirected. We refer to [11] for notation not defined here. Let $A(G)$ be the adjacent matrix of graph $G$. Since $A(G)$ is a real symmetric matrix, its eigenvalues must be real, and let $\lambda_{1}, \lambda_{2}, \ldots, \lambda_{n}$ denote the eigenvalues with $\lambda_{1} \geq \lambda_{2} \geq$ $\cdots \geq \lambda_{n}$. The sequence of $n$ eigenvalues of $A(G)$ is named the spectrum of $G$, and the spectral radius of $G$ often calls the largest eigenvalue $\lambda_{1}(G)$ and is denoted by $\rho(G)$. The characteristic polynomial of $A(G)$ is called the characteristic polynomial of the graph $G$ and is denoted by $\Phi(G)$. When $G$ is connected, $A(G)$ is an irreducible matrix. And by PerronFrobenius theorem [12], $\rho(G)$ is simple and the correspondent 
eigenvector can be positive. We refer to such eigenvector as Perron vector of $G$.

An internal path in a graph, denoted by $v_{0}, v_{1}, \ldots, v_{k}(k \geq$ 1 ), is a path joining vertices $v_{0}$ and $v_{k}$ which are both of degree greater than 3 , while all other vertices are of degree equal to 2 .

If graph $G$ with $n$ vertices has exactly two vertices of degree greater than two, then $G$ is double-star-like [13]. A double-star-like tree graph of order $n$ that has exactly two vertices with maximum degree 4 is called 4 -double-star-like tree, and it is denoted by $T_{4}^{2}(n)$. The degree sum of vertices adjacent to $v$ is called the second degree of $v$, remarked as $T\left(v_{i}\right)[4]$.

In this paper, we denote by $C_{n}$ and $T_{3}(n)$ the cycle with order $n$ and the third small spectral radius of tree. $\Gamma_{4}^{2}(n)$ denotes the family of the 4-double-star-like tree. Let $d(v)$ denote the degree of $v$. The sum of entries in the $i$ th row of $A$ is called $S_{i}(A)$.

Zhou and $\mathrm{Xu}[5]$ proved the following.

Let $G=(V, E)$ be a simple graph with $n$ vertices and $m$ edges, and then

$$
\begin{aligned}
& \rho(G) \leq\left(\Delta^{\prime}+\delta-1+\left(\left(\Delta^{\prime}+\delta-1\right)\right.\right. \\
& \left.\left.\quad+4\left(\Delta^{2}-\Delta \cdot \Delta^{\prime}+4 m-2(n-1) \delta\right)\right)^{1 / 2}\right) \times(2)^{-1}
\end{aligned}
$$

with equality if and only if $G$ is a regular bipartite graph.

$\mathrm{Wu}$ and $\mathrm{Hu}[10]$ gave a new upper bound of the spectral radii of $m$-star-like trees. They show the following result:

$$
\rho\left(G\left(S_{0}, S_{1}, S_{2}, \ldots, S_{\Delta_{0}}\right)\right)<\frac{(2 \Delta-1) \sqrt{2 \Delta-2}}{2 \Delta-2} .
$$

In this paper, we consider the extremal graphs and the upper bounds of the spectral radius of 4-double-star-like trees. The rest of the paper is organized as follows. Section 3 contains some useful lemmas which play a fundamental role. Section 4 contains three theorems on the 4-double-star-like trees.

\section{Some Useful Lemmas}

In this section, we use the following lemmas about the connected graph to prove our main results.

Lemma 1 (see [14]). Let uv be an edge of a connected graph $G$ with order $n$, and $G_{u, v}$ denote the graph obtained from $G$ by subdividing the edge uv by a new vertex $w$. Then the following two properties hold:

(1) If $u v$ is not on any internal path of $G \neq C_{n}$, then $\rho\left(G_{u, v}\right)>\rho(G)$.

(2) If $u v$ is on an internal path of $G \supsetneqq T_{3}(n)$, then $\rho\left(G_{u, v}\right)<$ $\rho(G)$.

Lemma 2 (see [15]). Let $v$ be a vertex of a connected graph $G$ with $d(v)>0$ and, for positive integers $i$ and $j$, let $G_{i, j}$ denote the graph got from $G$ by adding two pendent paths of length $i$ and $j$ at $v$. If $i \geq j \geq 1$, then $\rho\left(G_{i, j}\right)>\rho\left(G_{i+1, j-1}\right)$.

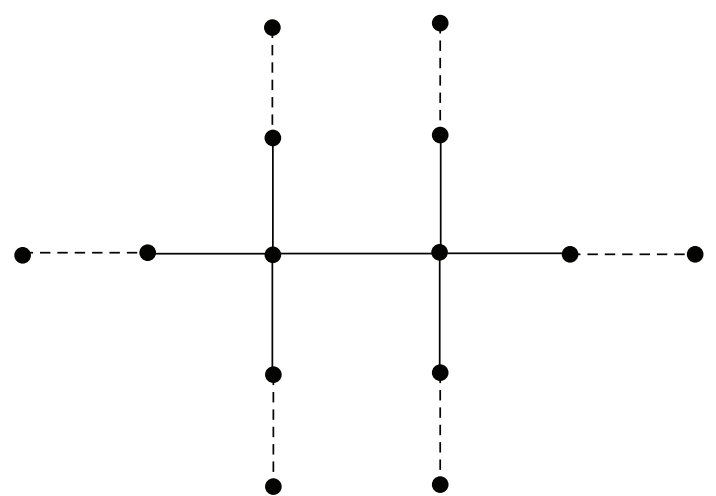

FIgURE 1: $T_{4}^{2}\left(i_{1}, i_{2}, i_{3}, 1, i_{4}, i_{5}, i_{6}\right)$.

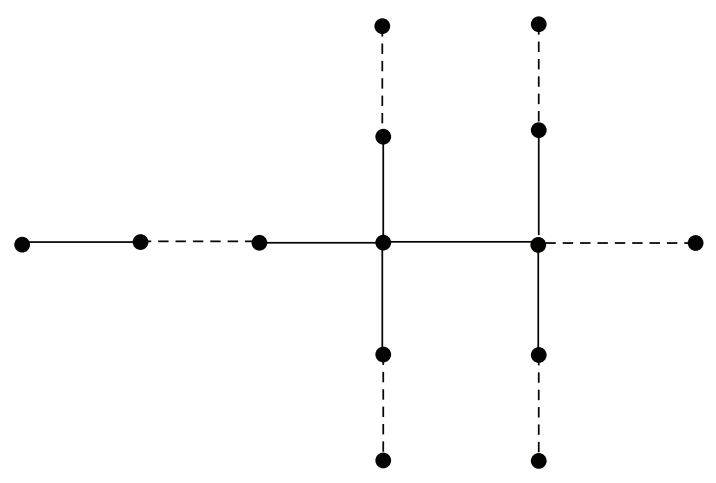

Figure $2: T_{4}^{2}\left(i_{1}+1, i_{2}, i_{3}, 1, i_{4}-1, i_{5}, i_{6}\right)$.

Lemma 3 (see [15]). Let $G$ be a connected graph with $a, b \in$ $E(G)$ and $\operatorname{deg} a>1$ and $\operatorname{deg} b>1$, and let $G_{i, j}^{(1)}$ be the graph obtained from $G$ by adding two pendent paths of $P_{i}$ and $P_{j}$ at $a, b$. If $i \geq j \geq 1$ and $x \geq \rho\left(G_{i+1, j-1}^{(1)}\right)$, then $\Phi\left(G_{i, j}^{(1)}, x\right)<$ $\Phi\left(G_{i+1, j-1}^{(1)}, x\right)$ and $\rho\left(G_{i, j}^{(1)}\right)>\rho\left(G_{i+1, j-1}^{(1)}\right)$.

From the above lemma we immediately get the following.

Corollary 4. Let $T_{4}^{2}(n)$ be a 4-double-star-like tree. As shown in Figure 1, when two centers are adjacent, $T_{4}^{2}(n)$ is denoted by $T_{4}^{2}\left(i_{1}, i_{2}, i_{3}, 1, i_{4}, i_{5}, i_{6}\right)$ with $i_{1}+i_{2}+i_{3}+i_{4}+i_{5}+i_{6}+2=n$ and $i_{1}>i_{4}$, where $i_{1}, i_{2}, i_{3}, i_{4}, i_{5}, i_{6}$ denote the length of the 6 branches of the 4-double-star-like tree in clockwise direction, respectively. Then, by the graft transformation, we get a graph, as shown in Figure 2 denoted by $T_{4}^{2}\left(i_{1}+1, i_{2}, i_{3}, 1, i_{4}-1, i_{5}, i_{6}\right)$. Then

$$
\begin{aligned}
& \rho\left(T_{4}^{2}\left(i_{1}, i_{2}, i_{3}, 1, i_{4}, i_{5}, i_{6}\right)\right) \\
& \quad>\rho\left(T_{4}^{2}\left(i_{1}+1, i_{2}, i_{3}, 1, i_{4}-1, i_{5}, i_{6}\right)\right) .
\end{aligned}
$$

Lemma 5 (see [1]). Let $G$ be a connected graph with order $n$ and spectral radius $\rho$ and $A$ denote its adjacency matrix. Let $f$ be any polynomial. Then

$$
\min _{v \in V(G)} S_{v}(f(A)) \leq f(\rho) \leq \min _{v \in V(G)} S_{v}(f(A)) .
$$




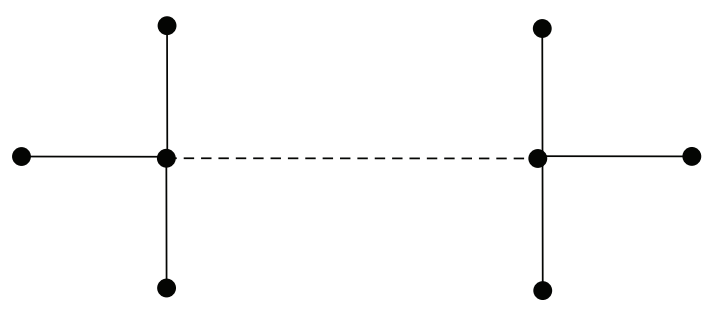

FIgURE 3: $T_{4}^{2}(1, n-6,1)_{1}$.

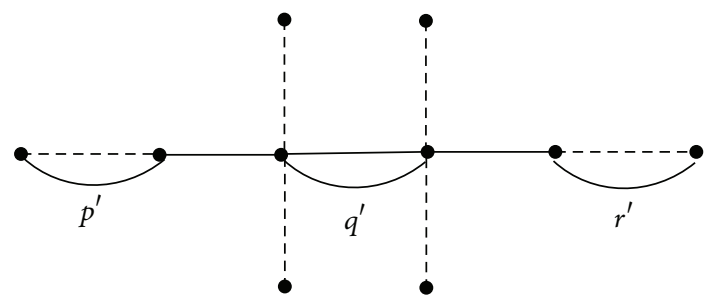

FIgURE 4: $T_{4}^{2}(n)$.

Furthermore, if the sum of all entries in each row of $f(A)$ are not all the same, then the inequalities are strict.

The following result provides a very effective method which can affect the value of spectral radius.

Lemma 6 (see [2]). Suppose $u, v$ are two vertices of a graph $G, u_{1}, u_{2}, \ldots, u_{s} \in N(u) \backslash N(v)(1 \leq s \leq d(u))$, and $x=\left(x_{1}, x_{2}, \ldots, x_{n}\right)$ is the Perron vector of $A(G)$, where $x_{i}$ corresponds to the vertex $u_{i}(1 \leq i \leq n)$. Let $G^{*}$ be the graph obtained from $G$ by deleting the edges $\left(u, u_{i}\right)$ and adding the edges $\left(v, u_{i}\right)(1 \leq i \leq s)$. If $x_{v} \geq x_{u}$, then $\rho(G)<\rho\left(G^{*}\right)$.

The processes depicted in Lemma 6 are called graft transformation.

\section{Main Results}

In this section, we consider 4-double-star-like tree with $n$ vertices. The main result of the paper is the following theorem.

Theorem 7. The minimal extremal graph of 4-double-star-like tree is $T_{4}^{2}(1, n-6,1)_{1}$ (as shown in Figure 3); namely,

$$
\rho\left(T_{4}^{2}(n)\right) \geq \rho\left(T_{4}^{2}(1, n-6,1)_{1}\right) .
$$

Proof. Let $T_{4}^{2}(n) \in \Gamma_{4}^{2}(n)$, as shown in Figure 4. By Lemma 2, we are using the graft transformation to obtain the graph, as shown in Figure 5, denoted by $T_{4}^{2}(n)_{1}$, and, then,

$$
\rho\left(T_{4}^{2}(n)\right) \geq \rho\left(T_{4}^{2}(n)_{1}\right) .
$$

Suppose $p^{\prime}, q^{\prime}$, and $r^{\prime}$ and $p, q$, and $r$ are positive integers. For the convenience of proof, we use $T_{4}^{2}\left(p^{\prime}, q^{\prime}, r^{\prime}\right)$ and $T_{4}^{2}(p, q, r)_{1}$ to denote $T_{4}^{2}(n)$ and $T_{4}^{2}(n)_{1}$, respectively. $p^{\prime}$, $q^{\prime}$, and $r^{\prime}$ denote the number of the vertices of three parts as

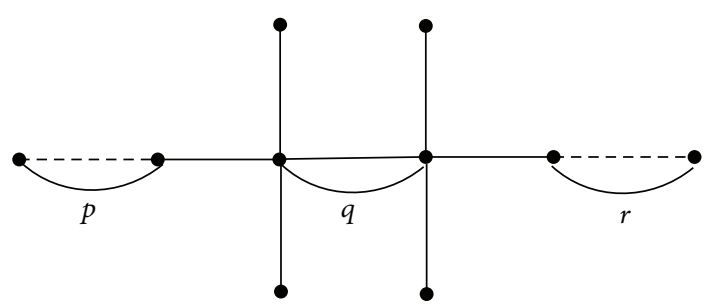

Figure 5: $T_{4}^{2}(n)_{1}$.

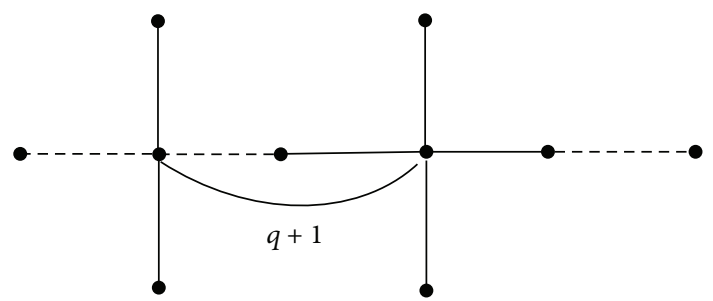

Figure 6: $T_{4}^{2}(p-1, q+1, r)_{1}$.

shown in Figure 4. Similarly, $p, q$, and $r$ play the same role in Figure 5.

Obviously, $T_{4}^{2}(p, q, r)_{1}$ is a subdivision of external of $T_{4}^{2}(p-1, q, r)_{1}$, and then, by using Lemma 1 , we have

$$
\rho\left(T_{4}^{2}(p, q, r)_{1}\right) \geq \rho\left(T_{4}^{2}(p-1, q, r)_{1}\right) .
$$

As shown in Figure 6, $T_{4}^{2}(p-1, q+1, r)_{1}$ is a subdivision of internal $T_{4}^{2}(p-1, q, r)_{1}$, and, by Lemma 1 , we have

$$
\rho\left(T_{4}^{2}(p-1, q, r)_{1}\right) \geq \rho\left(T_{4}^{2}(p-1, q+1, r)_{1}\right) .
$$

By (7) and (8),

$$
\rho\left(T_{4}^{2}(p, q, r)_{1}\right) \geq \rho\left(T_{4}^{2}(p-1, q+1, r)_{1}\right) .
$$

Proceeding in this way, we have

$$
\begin{gathered}
\rho\left(T_{4}^{2}(p-1, q+1, r)_{1}\right) \geq \rho\left(T_{4}^{2}(p-2, q+2, r)_{1}\right) \\
\rho\left(T_{4}^{2}(p-2, q+2, r)_{1}\right) \geq \rho\left(T_{4}^{2}(p-3, q+3, r)_{1}\right) \\
\vdots \\
\rho\left(T_{4}^{2}(1, p+q-1, r)_{1}\right) \\
\geq \rho\left(T_{4}^{2}(1, p+q+r-2,1)_{1}\right) .
\end{gathered}
$$

Thus

$$
\begin{aligned}
\rho\left(T_{4}^{2}(p, q, r)_{1}\right) & \geq \rho\left(T_{4}^{2}(1, p+q-1, r)_{1}\right) \\
& \geq \rho\left(T_{4}^{2}(1, p+q+r-2,1)_{1}\right) .
\end{aligned}
$$

As shown in Figure $7, T_{4}^{2}(1, p+q+r-2,1)$ is the minimum extremal graphs of 4 -double-star-like tree, and $p+q+r-2=$ $n-6$, shortened to $T_{4}^{2}(1, n-6,1)_{1}$. 


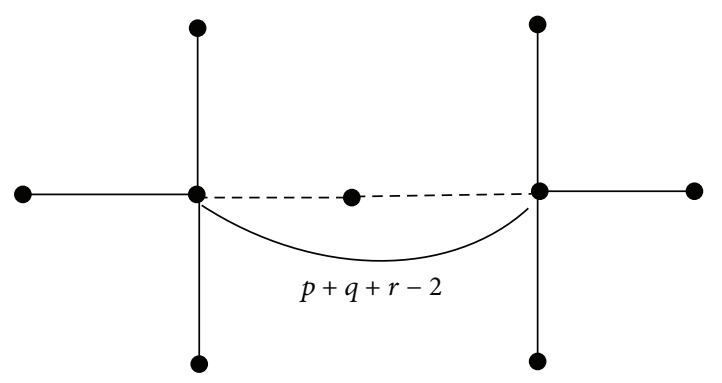

Figure $7: T_{4}^{2}(1, p+q+r-2,1)_{1}$.

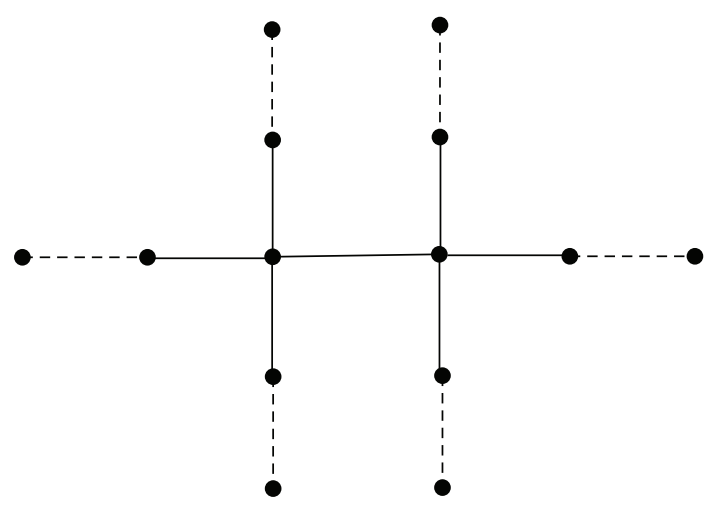

FiguRE 8: $T_{4}^{2}\left(a_{1}, a_{2}, a_{3}, 1, a_{4}, a_{5}, a_{6}\right)$.

Theorem 8. The maximum extremal graph of 4-double-starlike tree is $T_{4}^{2}\left(a_{1}, a_{2}, a_{3}, 1, a_{4}, a_{5}, a_{6}\right)$ (as shown in Figure 8), where $a_{1}, a_{2}, \ldots, a_{6}$ are almost equal in length; namely, the length of each branch differs at most 1 :

$$
\rho\left(T_{4}^{2}(n)\right) \leq \rho\left(T_{4}^{2}\left(a_{1}, a_{2}, a_{3}, 1, a_{4}, a_{5}, a_{6}\right)\right) .
$$

Proof. Let $T_{4}^{2}(n) \in \Gamma_{4}^{2}(n)$, as shown in Figure 9.

Obviously, $T_{4}^{2}(p, q, r)$ is a subdivision of internal $T_{4}^{2}(p, q-$ $1, r)$, and, by Lemma 1 , we can get

$$
\rho\left(T_{4}^{2}(p, q-1, r)\right) \geq \rho\left(T_{4}^{2}(p, q, r)\right) .
$$

Then, as shown in Figure 10, $T_{4}^{2}(p+1, q-1, r)$ is a subdivision of external $T_{4}^{2}(p, q-1, r)$, and, by Lemma 1 , we can get

$$
\rho\left(T_{4}^{2}(p+1, q-1, r)\right) \geq \rho\left(T_{4}^{2}(p, q-1, r)\right) .
$$

By (13) and (14), we can get

$$
\rho\left(T_{4}^{2}(p+1, q-1, r)\right) \geq \rho\left(T_{4}^{2}(p, q, r)\right) .
$$

Proceeding in this way until $q=2$, we get $T_{4}^{2}(p+q-2,2, r)$, which can be denoted in the form of $T_{4}^{2}\left(i_{1}, i_{2}, i_{3}, 1, i_{4}, i_{5}, i_{6}\right)$ as in Corollary 4. The value of spectral radius is largest when $i_{1}, i_{2}, i_{3}, i_{4}, i_{5}, i_{6}$ equal $a_{1}, a_{2}, a_{3}, a_{4}, a_{5}, a_{6}$ and $a_{1}, a_{2}, \ldots, a_{6}$ are almost equal in length, and then we get $T_{4}^{2}\left(a_{1}, a_{2}, a_{3}, 1, a_{4}, a_{5}, a_{6}\right)$, as shown in Figure 11 .

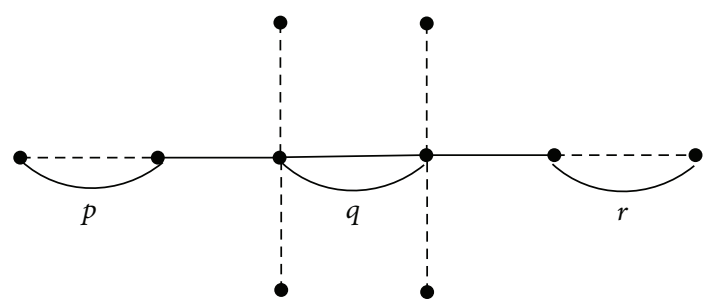

Figure 9: $T_{4}^{2}(n)$.

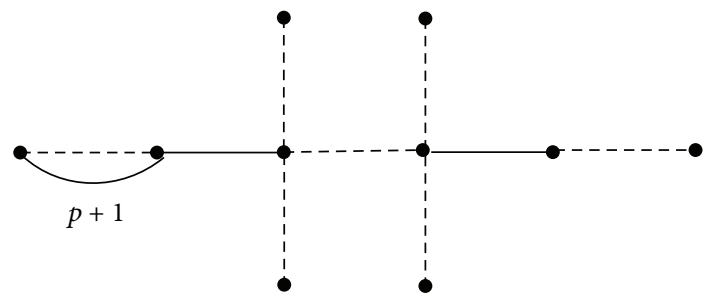

Figure 10: $T_{4}^{2}(p+1, q-1, r)$.

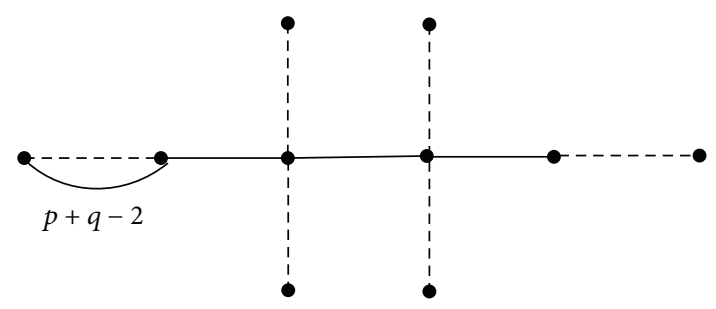

FIGURE 11: $T_{4}^{2}(p+q-2,2, r)$.

When $n=11$, there are 6 different 4 -double-star-like trees (as shown in Figure 12). By calculating the spectral radius of the 6 graphs, we can see that Theorem 8 holds for the 6 graphs:

$$
\begin{aligned}
& \rho(T(1,5,1))=2.1753, \\
& \rho(T(1,4,2))=2.2262, \\
& \rho(T(1,3,3))=2.2738, \\
& \rho(T(1,2,4))=2.3459, \\
& \rho(T(2,2,3))=2.3700, \\
& \rho(T(2,3,2))=2.2882,
\end{aligned}
$$

and then

$$
\begin{aligned}
\rho(T(2,2,3)) & >\rho(T(1,2,4))>\rho(T(2,3,2)) \\
& >\rho(T(1,3,3))>\rho(T(1,4,2)) \\
& >\rho(T(1,5,1)) .
\end{aligned}
$$

Theorem 9. The upper bound of spectral radius of the 4double-star-like trees is $1+\sqrt{5}$; namely,

$$
\rho(T(j, k, l, m)) \leq 1+\sqrt{5} \text {. }
$$




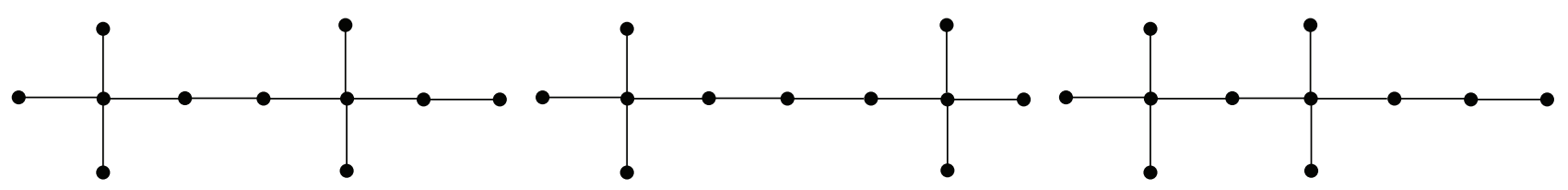

$T_{4}^{2}(1,4,2)$

$T_{4}^{2}(1,5,1)$

$T_{4}^{2}(1,3,3)$

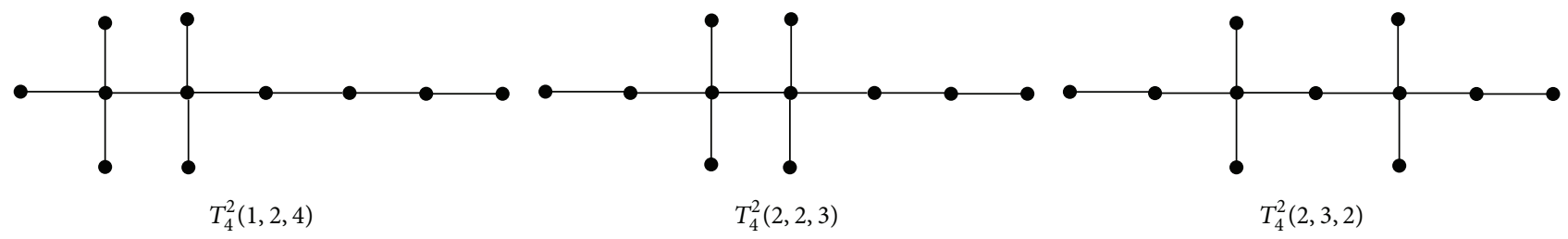

Figure 12: $T_{4}^{2}(11)$.

Proof. By the definition of 4-double-star-like tree we can get

$$
\begin{gathered}
T\left(v_{i}\right)-2 d\left(v_{i}\right)=2, \\
T\left(v_{j}\right)-2 d\left(v_{j}\right)=0, \\
T\left(v_{k}\right)-2 d\left(v_{k}\right)=-1, \\
T\left(v_{m}\right)-2 d\left(v_{m}\right)=-3, \\
T\left(v_{n}\right)-2 d\left(v_{n}\right)=4 .
\end{gathered}
$$

Let $A$ be the adjacent matrix of $T_{4}^{2}(n)$. Since the sum of entries in the $i$ th row of $A$ is $d\left(v_{i}\right), S_{i}(A)=d\left(v_{i}\right)$. Since the sum of entries in the $i$ th row of $A^{2}$ is $T\left(v_{i}\right), S_{i}\left(A^{2}\right)=T\left(v_{i}\right)$.

By (19) we obtain

$$
S_{i}\left(A^{2}\right)-2 \times S_{i}(A) \leq 4 .
$$

Suppose $f(x)=x^{2}-2 x$. By Lemma 5 , we have

$$
\rho^{2}-2 \rho \leq 4
$$

Thus

$$
\rho \leq 1+\sqrt{5}
$$

Now, we complete the proof of Theorem 9.

\section{Conclusion}

In this paper we have shown some results of the 4-doublestar-like trees. We obtain the minimal and the maximum extremal graphs of 4 -double-star-like tree being $T_{4}^{2}(1, n-$ $6,1)_{1}$ and $T_{4}^{2}\left(a_{1}, a_{2}, a_{3}, 1, a_{4}, a_{5}, a_{6}\right)$, and then we get the upper bound of spectral radius of the 4 -double-star-like trees, which is $1+\sqrt{5}$.

\section{Conflict of Interests}

The authors declare that there is no conflict of interests regarding the publication of this paper.

\section{Acknowledgments}

This research is supported by the National Natural Science Foundation of China (11426125 and 61473139), Program for Liaoning Excellent Talents in University LR2014016, and the Educational Commission of Liaoning Province (L2014239).

\section{References}

[1] M. N. Ellingham and X.-Y. Zha, "The spectral radius of graphs on surfaces," Journal of Combinatorial Theory Series B, vol. 78, no. 1, pp. 45-56, 2000.

[2] X.-X. Wang, M.-Q. Zhai, and J.-L. Shu, "Upper bounds on the spectral radius of k trees," Applied Mathematics-A Journal of Chinese Universities, vol. 26, no. 9, pp. 209-214, 2011.

[3] H.-L. Gong, "Some sharp lower bounds for spectral radius of connected graphs," Journal of East China Normal University, vol. 4, no. 5, pp. 18-26, 2012.

[4] J.-S. Yuan and J.-L. Shu, "A new upper bound for the spectral radius of Halin graphs and the extremal graphs," Applied Mathematics-A Journal of Chinese Universities, vol. 23, no. 33, pp. 335-342, 2008.

[5] H. Zhou and Y. Xu, "Sharp upper bounds for the Laplacian spectral radius of graphs," Mathematical Problems in Engineering, vol. 2013, Article ID 720854, 4 pages, 2013.

[6] H. Z. Song and Q. F. Wang, "Properties and applications of the eigenvector corresponding to the laplacian spectral radius of a graph," Mathematical Problems in Engineering, vol. 2013, Article ID 524162, 9 pages, 2013.

[7] H.-Z. Song, Q.-F. Wang, and L.-L. Tian, "New upper bounds on the spectral radius of trees with the given number of vertices and maximum degree," Linear Algebra and Its Applications, vol. 439, no. 9, pp. 2527-2541, 2013.

[8] L. Patuzzi, M. A. A. de Freitas, and R. R. Del-Vecchio, "Indices for special classes of trees," Linear Algebra and its Applications, vol. 442, pp. 106-114, 2014.

[9] G. Aalipour, S. Akbari, and N. Shajari, "Laplacian spectral characterization of two families of trees," Linear and Multilinear Algebra, vol. 62, no. 7, pp. 965-977, 2014.

[10] T.-Z. Wu and S.-B. Hu, "On the spectral radii of m-starlike tree," Operations Research Transactions, vol. 15, no. 3, pp. 45-50, 2011.

[11] J. A. Bondy, Graph Theory with Applications, Elsevier, New York, NY, USA, 1st edition, 1976. 
[12] B. Liu, Combinatorial Matrix Theory, Science Press, Beijing, China, 2nd edition, 2005.

[13] P.-L. Lu and Z.-T. Yuan, "Double-starlike tree $H_{n}(p, 2)$ determined by its Lapiacian spectrum," Journal of Lanzhou University of Technology, vol. 34, no. 6, pp. 364-369, 2008.

[14] D. M. Cvetković and P. Rowlinson, "The largest eigenvalue of a graph: a survey," Linear and Multilinear Algebra, vol. 28, no. 1-2, pp. 3-33, 1990.

[15] X.-D. Chen, "Further ordering trees with perfect matchings by their spectral radius," Journal of Xiamen University (Natural Science), vol. 52, no. 11, pp. 297-301, 2013. 


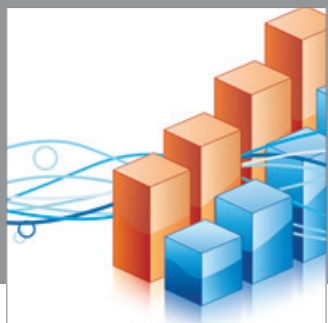

Advances in

Operations Research

mansans

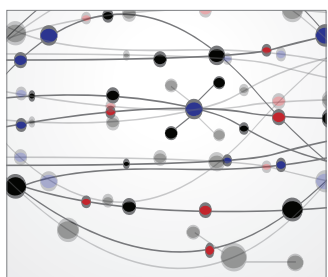

The Scientific World Journal
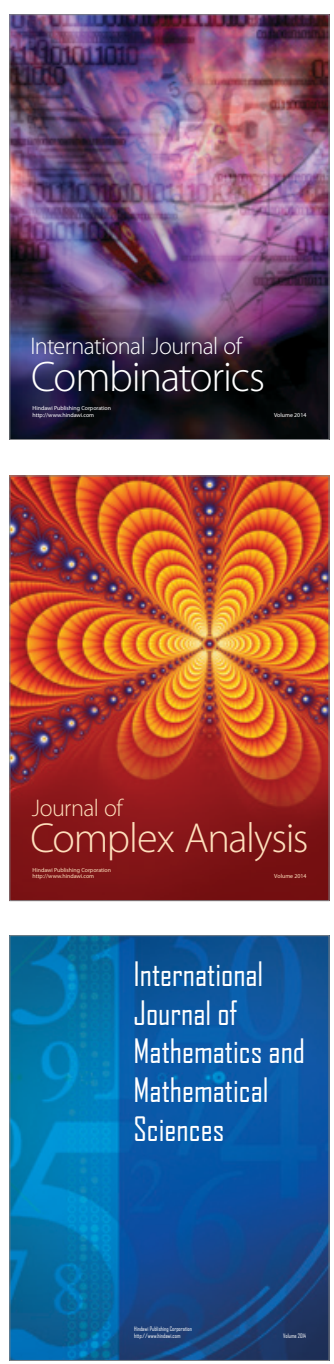
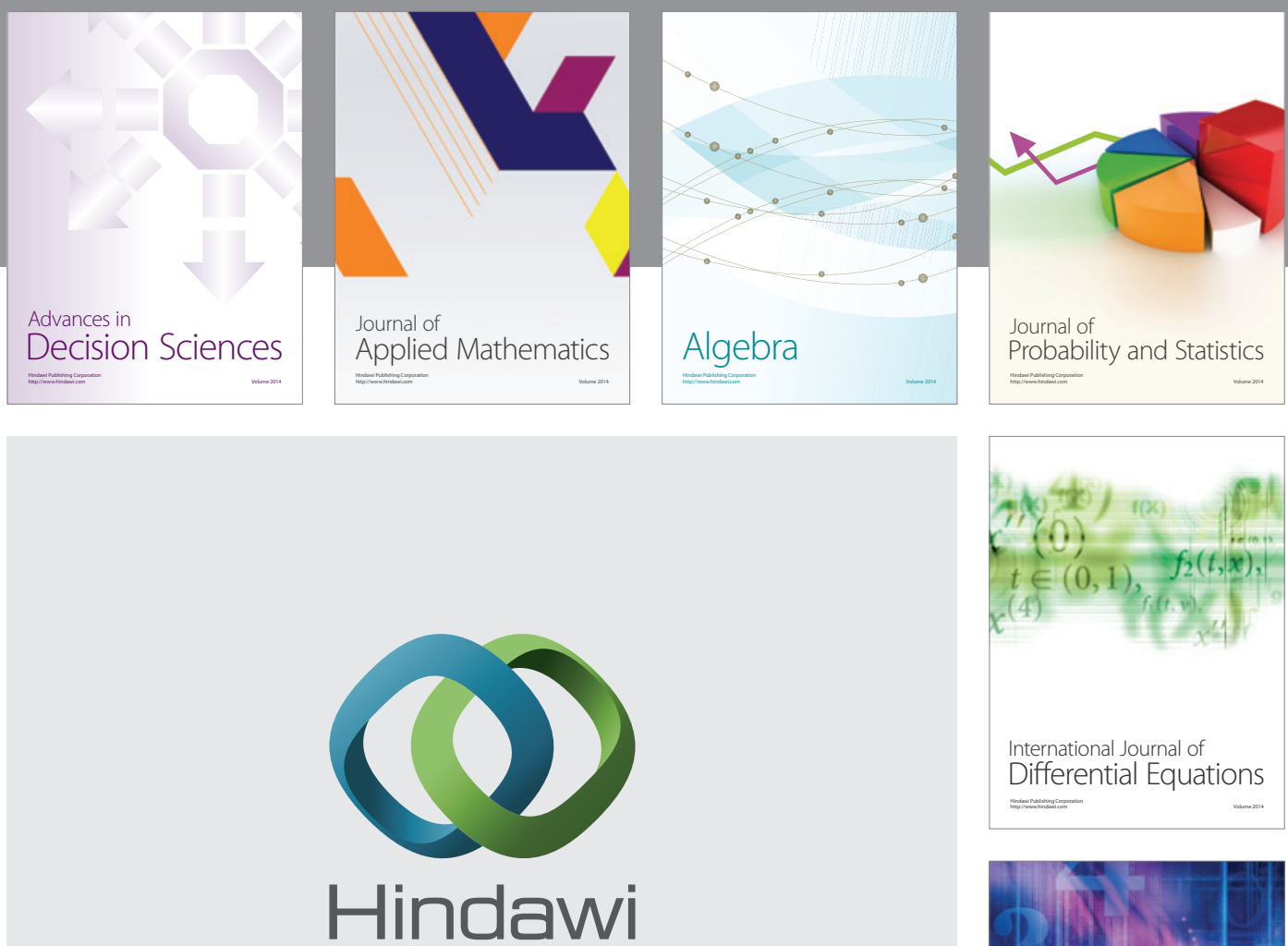

Submit your manuscripts at http://www.hindawi.com
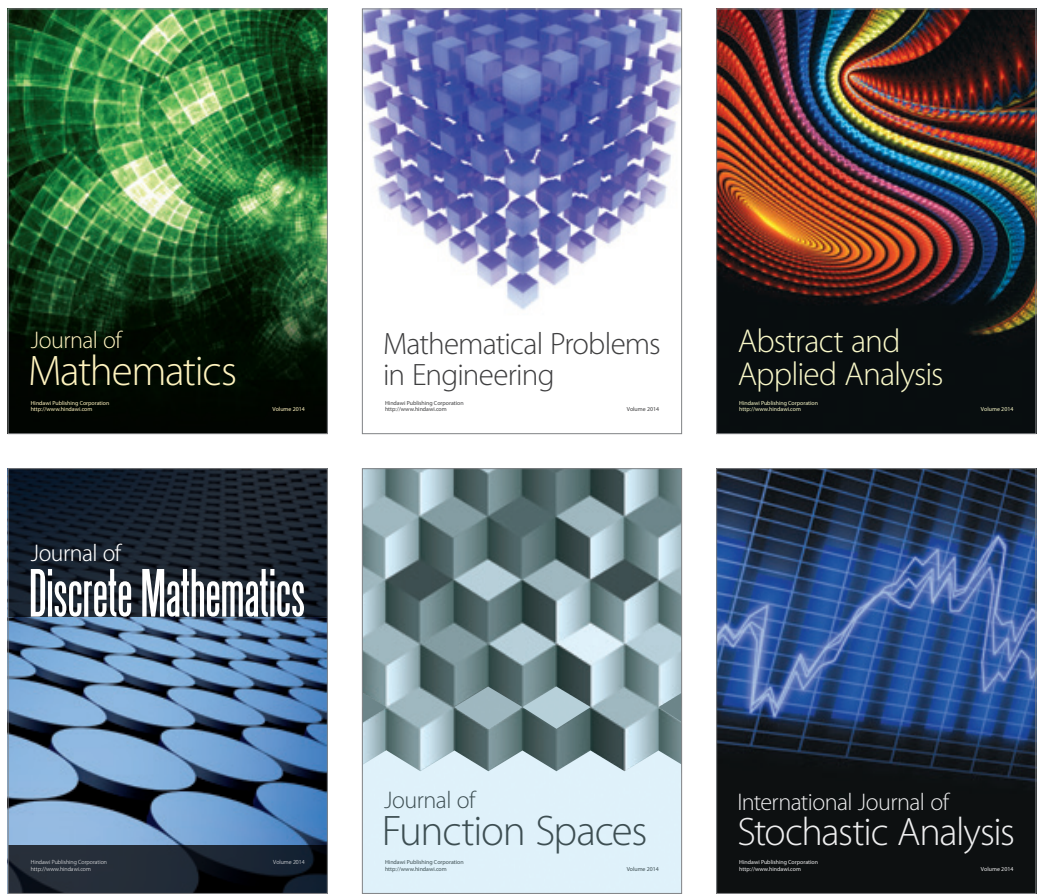

Journal of

Function Spaces

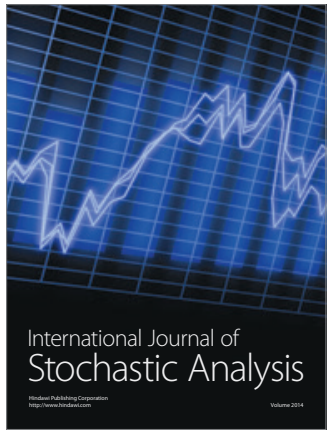

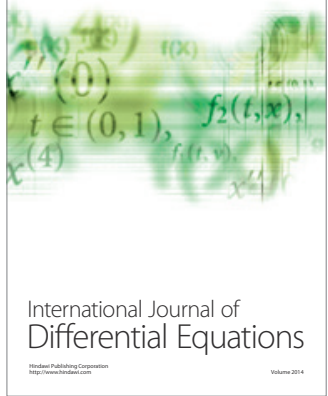
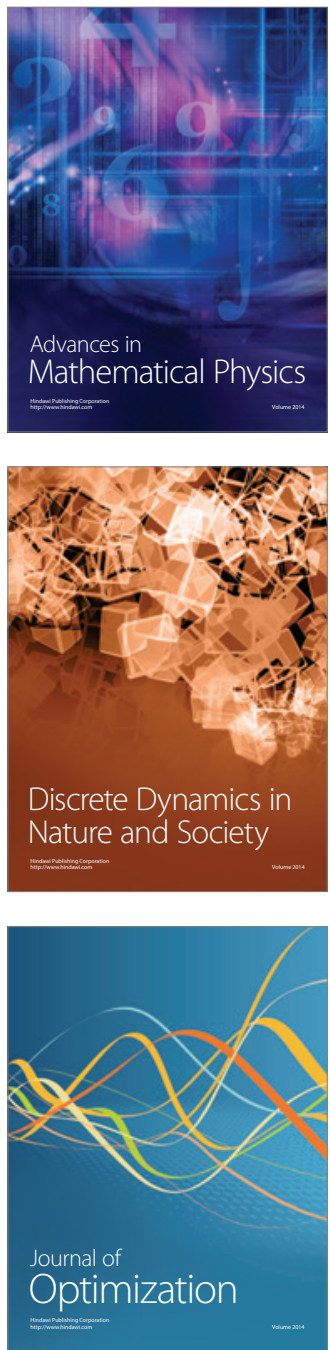